\title{
Uso de las TIC, Internet y redes sociales durante la campaña electoral exterior. Caso de las elecciones de los diputados de ultramar de la República Dominicana del $2012^{1}$
}

\section{Use of ICT, Internet and social networks during the external electoral campaign. Case of the 2012 Dominican Republic overseas deputies elections}

Dra. Kenia R. Del Orbe Ayala

Profesora Asociada Departamento de Ciencias de la Comunicación y Sociología (Universidad Rey Juan Carlos)

Fecha de recepción: 15 de octubre de 2017

Fecha de revisión: 8 de junio de 2018

Fecha de publicación: 1 de julio de 2018

Para citar este artículo: Del Orbe Ayala, K. R. (2018). Uso de las TIC, Internet y redes sociales durante la campaña electoral exterior. Caso de las elecciones de los diputados de ultramar de la República Dominicana del 2012, Icono 14, 16 (2), 1-26. doi: 10.7195/ri14. v16i2.1122 


\section{Resumen}

Actualmente la Tecnologías de la Información y la Comunicación (TIC), Internet y las redes sociales están jugando un papel importante en el campo de la comunicación política electoral. En este artículo estudiamos su aplicación dentro de las campañas electorales. Nos planteamos como objetivo conocer el uso que ofrecieron a las citadas herramientas digitales los candidatos a diputados de ultramar de la República Dominicana que participaron en las elecciones del año 2012. Utilizamos la metodología cuantitativa a través de la técnica de investigación de la encuesta que realizamos a los encargados de comunicación de los postulantes. Los resultados arrojan: el uso pasivo que realizaron los candidatos a diputados de ultramar en cuanto a inversión publicitaria se refiere desde el entorno digital, las dos redes sociales más utilizadas en la campaña fueron Facebook y YouTube, hubo candidatos que prefirieron no utilizar páginas webs o blogs. La red social mejor valorada por las candidaturas fue Facebook y la peor valoradora LinkedIn. En conclusión descubrimos que una gran parte de los candidatos no realizó un uso estratégico de las TIC, Internet, y las redes sociales en la campaña electoral.

Palabras clave: Comunicación política - Diputados de ultramar - República Dominicana - Redes sociales - TIC - Internet

\section{Abstract}

Currently Information and Communication Technologies (ICT), Internet and social networks are playing an important role in the field of electoral politics communication. In this article we study its application within electoral campaigns. We intend to know how the use of the digital tools offered to the candidates to overseas deputies of the Dominican Republic who participated in the elections of the year 2012. We use the quantitative methodology through the investigation technique of the survey that we conducted to the communication agents of the postulants. The results show that the passive use of candidates for overseas deputies in terms of advertising investment refers to the digital environment, the two most used social networks in the campaign were Facebook and YouTube, there were candidates who preferred not to use websites or blogs. The social network best valued by the candidacies was Facebook and the 
Uso de las TIC, Internet y redes sociales durante la campaña electoral exterior... | 3 ARTÍCULOS DE INVESTIGACIÓN

worst valued was LinkedIn. In conclusion, we discovered that a large number of candidates did not make strategic use of ICTs, the Internet, and social networks in the electoral campaign.

Key Words: Political communication - Deputies - Dominican Republic - Social networks - ICT - Internet

\section{Introducción. Elecciones de los diputados de ul- tramar de la República Dominicana del 2012. Una mirada hacia el estudio del entorno digital}

La aplicación de la Tecnologías de la Información y la Comunicación (TIC), Internet y las redes sociales durante las campañas electorales, cada día ofrecen más posibilidades desde el uso estratégico de la comunicación política, gracias a las ventajas que ofrecen tanto a los políticos como a los ciudadanos, por lo que definitivamente podríamos decir que han transformado todo el ecosistema mediático, político y social.

Con relación al uso de las TIC, Internet y las redes sociales, utilizadas como nuevas formas de comunicación digital vemos que:

Las posibilidades de interconectividad e hiperconectividad propiciadas por estas plataformas de comunicación han cambiado las formas de relacionarnos, de compartir conocimiento, de formarnos e informarnos. En esta era de la no desconexión (END) curioso (acrónimo), las tecnologías, los buscadores, las redes sociales, los operadores han traído consigo un cambio que nos permite hablar, incluso, de una nueva forma de ciudadanía, la denominada ciudadanía digital. (García y Fernández, 2016: IX).

Como vemos el uso del Internet ha transformado la forma de comunicación de las sociedades. En ese sentido, en la investigación que presentamos sobre la representación política parlamentaria exterior con los diputados de ultramar, analizamos a nivel de comunicación política el entorno digital desde las estrategias de los candidatos que participaron en el proceso. 
A continuación en este apartado introductorio resumimos la experiencia de la República Dominicana con las elecciones de los diputados de ultramar. Desde la visión de América Latina, conoceremos cuáles países de Latinoamérica incluyen este tipo de representación.

Hasta la fecha existen unos 150 países que contemplan regulaciones relacionadas a permitir el voto desde el extranjero de sus nacionales radicados en el exterior (Navarro, 2016). Aunque en la medida que pasan los años la cifra va en aumento, "hasta la fecha, los trabajos académicos sobre el voto en el exterior se han concentrado principalmente en cuestiones normativas (López-Guerra, 2005; Rubio-Marín, 2006, Baubock, 2007). (En Lafleur, 2012: 18). (Citado por Del Orbe, 2017:75).

Como una forma de hacer frente a la citada limitación desde el ámbito de estudio del voto en el exterior, en el presente trabajo se estudia el uso de las TIC, Internet y las redes sociales por parte de los candidatos que participaron en las elecciones de los diputados de ultramar de la República Dominicana del 2012.

Los diputados de ultramar dominicanos son siete legisladores que representan a la comunidad dominicana en el exterior, divididos en tres circunscripciones. La primera circunscripción correspondiente a Canadá y Estados Unidos, en la que se asignan 3 diputados; la segunda abarca parte de El Caribe, Latinoamérica y Miami, con 2 diputados; y la tercera le corresponde a Europa, con 2 diputados también.

Aunque se presentó un impedimento para celebrar las elecciones del 2012 en Canadá, pues semanas antes de realizarse la contienda electoral, el gobierno canadiense, haciendo uso del principio de soberanía internacional, ordenó solo permitir las votaciones a nivel presidencial y no así para los diputados de ultramar (De León, 2012).

Además de República Dominicana, existen dos países en América Latina que incluyen la modalidad del voto destinado a elegir representantes ante el parlamento del país origen de sus nacionales radicados en el exterior (Colombia y Ecuador). 
Uso de las TIC, Internet y redes sociales durante la campaña electoral exterior... | 5 ARTÍCULOS DE INVESTIGACIÓN

\begin{tabular}{|c|c|c|}
\hline País & Escaños & \% del total \\
\hline Colombia & 1 de 166 en la Cámara Baja & $0.60 \%$ \\
\hline Ecuador & $\begin{array}{c}6 \text { de } 137 \text { en la Asamblea } \\
\text { Nacional }\end{array}$ & $4.37 \%$ \\
\hline República Dominicana & 7 de 190 en la Cámara Baja & $3.68 \%$ \\
\hline
\end{tabular}

Tabla 1: Países de América Latina con representación parlamentaria de sus ciudadanos en el extranjero. Fuentes: elaboración propia a partir de datos de (Navarro et al., 2007; Navarro, 2012 y Asamblea Nacional de Ecuador, 2016).

La República Dominicana entró en la vanguardia de este tipo de elecciones a nivel de ejecución el 20 de mayo del 2012 con la celebración de las elecciones de los diputados de ultramar, la cual se realizó en conjunto con las elecciones presidenciales. De manera que dicho proceso representó una transformación en el sistema político- electoral dominicano, al ser elegidos por primera vez en la historia del país la República Dominicana los legisladores que representan a la comunidad dominicana en el extranjero.

El tema de las elecciones de los diputados de ultramar de la República Dominicana, al ser una transformación reciente en el sistema político electoral dominicano, hemos comprobado que no ha sido tratado en profundidad a nivel científico. De las investigaciones exploradas: (Vargas, 2011a; Vargas, 2011b; Vargas, 2012; Gautreau, 2012; Sepúlveda, 2012; JCE, 2013; y 0EA, 2013). Observamos que varios de los textos citados fueron publicados en la página web del Observatorio Político Dominicano. Dicho portal es una iniciativa de la Fundación Global Democracia y Desarrollo (FUNGLODE), presidida por el expresidente de la República Dominicana, Leonel Fernández, y quien hasta el momento de redacción de este artículo seguía siendo presidente del Partido de la Liberación Dominicana (PLD), partido que asume la presidencia de la República.

Por otra parte podríamos decir que las investigaciones tienen en común que no profundizan mucho en el tema de los diputados de ultramar. Se tratan de textos breves o conferencias que en su gran mayoría no pasan de 5 páginas y se centran, sobre todo en analizar el padrón electoral en el exterior y la participación electoral. 
Como es sabido en el campo de la investigación en comunicación política, los investigadores se han focalizado más en estudiar los procesos de elecciones (Mazzoleni, 2004). “Una campaña electoral trata de mover los votos que necesitamos para ganar, a través de la comunicación orientada al elector" (Elgarresta, 2003: 17).

\section{Internet, TIC y redes sociales en las campañas electorales}

Para el ámbito de la política Internet representa una herramienta joven, tuvieron que pasar muchos años a partir de la llegada del primer registro inicial sobre el “(...) hipertexto en 1991, que los actores políticos se animaron a probar, en forma renuente, las capacidades de Internet (...) sería otro Kennedy y de nombre Eduard, quien, a la postre se convertiría en el primer político virtual de la historia" (Galeas de la Vega, 2012: 204).

Antes de Internet alcanzar el gran auge que tiene en la actualidad, el investigador Manuel Castells (2003) explicaba que era una creación cultural como pasa con la creación de toda tecnología, tanto así que, "al ser una tecnología de comunicación interactiva con fuerte capacidad de retroacción, los usos de Internet se plasman en su desarrollo como red y en el tipo de aplicaciones tecnológicas que van surgiendo" (2003: 4).

Para el caso de las campañas electorales, tema que analizamos aquí, Internet otorga agilidad y rapidez a la campaña, ya que permite manejar, adquirir y gestionar un gran volumen de información en muy poco tiempo. Lo que obliga a contar con un personal especializado en la materia (Elgarresta, 2003).

Internet, aparte de ser utilizada como una herramienta publicitaria para promocionar y difundir el mensaje electoral, también se utiliza para recibir donaciones económicas destinadas a la financiación de las campañas. Un ejemplo reciente y muy popular de dicha práctica lo vimos en las elecciones generales españolas del 2016, fue el caso del partido de Podemos, que utilizó una forma muy peculiar para financiar su campaña "a través de donaciones y microcréditos de sus simpatizantes (...) a través de las redes, con el hashtag \#YoNoTengounBárcenas (haciendo refe- 
Uso de las TIC, Internet y redes sociales durante la campaña electoral exterior... | 7

rencia al ex tesorero corrupto del Partido Popular), y con una página web específica de donaciones donde se explicaba cuánto se iba consiguiendo" (Gutiérrez-Rubí, 2016). Otros casos de éxitos en recaudación de fondos por Internet que destaca Gutiérrez-Rubí son: el del partido español Ciudadanos en la misma campaña del 2016, en el último trimestres del 2015 el caso del candidato demócrata Bernie Sanders en Estados Unidos, que obtuvo 26 millones de dólares en donaciones, Obama durante la campaña del 2008 y 2012, entre otras campañas de crowdfunding, término con el que se le suele llamar al citado método de recaudación de fondos.

En relación al uso del Internet, como ventaja principal en comparación con otros medios tradicionales, se puede decir, que es un medio mucho más económico para insertar publicidad. En la actualidad el uso de las TIC está jugando un papel importante en la comunicación política dentro de las campañas electorales, gracias a las ventajas que ofrecen tanto a los políticos como a los ciudadanos. Desde las perspectivas descritas, podemos decir que definitivamente han transformado todo el ecosistema mediático. Aunque a pesar de las ventajas que ofrecen muchos políticos se mantienen rezagados en cuanto a su uso.

El tema del momento en los congresos, cumbres sobre comunicación política, artículos e investigaciones científicas y publicaciones en general, lo han estado protagonizando el uso de las nuevas tecnologías y las redes sociales en la comunicación política, específicamente en campañas electorales. Al respecto veamos algunas posiciones de expertos en la materia:

Crespo en el marco del IV Congreso Uruguayo de Ciencia Política decía que "las nuevas tecnologías de la información y la comunicación, y más recientemente la aparición de las redes sociales, están cambiando el perfil de la comunicación de campaña" (Mieres, 2015: 1). En cambio, con una posición más crítica y optimista, se sitúa Mario Riorda, al afirmar que la comunicación política de hoy "va adquiriendo un fuerte influjo tecnológico que independientemente de ser o no una herramienta central, va produciendo cambios sustantivos en las prácticas de la política. Y como la comunicación, vía el impacto tecnológico, está mutando aceleradamente, muta aceleradamente la política" (Mieres, 2015: 1). Por su parte Daniel Ureña reconoce que, “las nuevas tecnologías están cambiando la política. La forma 
en que ciudadanos y políticos se comunican entre sí está en continua evolución gracias a Internet. Nuevos códigos, lenguajes y canales se han incorporado en los últimos años" (2011: 30). Alfredo Dávalos explica que actualmente "las campañas tienen la posibilidad de disponer de nuevas tecnologías a través de poderosas herramientas como Internet y el celular, los cuales han sido partícipes en distintos hechos históricos y políticos, logrando incluso, movilizar e impactar a nivel mundial (2011: 2).

Los consultores e investigadores antes citados (Ureña, 2011; Dávalos, 2011; Crespo y Riorda, en Mieres, 2015), coinciden en sus planteamientos sobre el uso de Internet y las TIC en el campo de la comunicación política, al afirmar que la forma de hacer comunicación política ha cambiado gradualmente, tanto para los políticos como para los ciudadanos. Cabe considerar que ese cambio no sólo se ha limitado a los políticos y a los ciudadanos, sino, más bien se convierte en un trípode, ya que también ha abarcado a los medios de comunicación tradicionales, ya que se han visto en la necesidad de tener que adaptar sus formatos.

Ahora bien, un ejemplo clásico del uso de Internet y las TIC en los procesos de campañas electorales, fue la campaña presidencial del 2008 de Barack Obama en Estados Unidos, quien logró conectar con los votantes gracias al buen uso de las TIC. Algo semejante, se volvió a repetir en la campaña presidencial del 2012 con la reelección. La comunicación política del presidente Obama ha marcado un antes y un después.

Castro explica que Obama representa, "no sólo el mejor ejemplo de Estados Unidos, sino que quizás a nivel internacional de lo que la Internet, el correo electrónico, los celulares, los blogs, etc., pueden hacer para lograr la empatía con la gente y lograr adeptos" (2012: 209). En una tesitura parecida a la de Castro se sitúan los investigadores D’Adamo, García y Kievsky, quienes afirman que "lo sucedido con Obama en 2008 causó tal impresión en el mundo que "Obama" se convirtió prácticamente en sinónimo de campaña digital o "elecciones 2.0" (2015: 108). No podemos decir que Barack Obama fue lo mejor de USA, pero de lo que sí estamos claros es que revolucionó la comunicación política del siglo XXI. 
Uso de las TIC, Internet y redes sociales durante la campaña electoral exterior... | 9

Sobre el uso de las campañas digitales Rishad Tobaccowala, especialista en temas de publicidad digital, enumeró cuatro causas de por qué el presidente Obama se alzó con la nominación demócrata: en primer lugar, por la magnífica excelencia en el manejo de campaña por Internet. En segundo lugar, por el empleo del correo electrónico. Tercero, por la realización de llamativos videos musicales. Y, por último, gracias a las nuevas tecnologías que le permitieron expandir sus mensajes (Valdez y Huerta, 2008: 5, citado por Castro, 2012).

Con el uso de las redes sociales en las campañas políticas, cada vez más los candidatos hacen un uso inteligente y estratégico de las principales redes sociales durante las campañas electorales, dichas herramientas les permiten un contacto cercano con el público e interactuar sobre diferentes temas, aunque la mayoría solo decidan usarla para compartir información electoral con sus seguidores, y no así para responder a sus inquietudes.

En ese mismo sentido Miguel Túñez y José Sixto explican que:

La política 2.0 también requerirá la capacidad del político para abrirse a los ciudadanos, fomentando su participación y valorando sus comentarios u opiniones. La creación de los blogs o perfiles en redes sociales que solo patenten presencia y no participación provocarán en la audiencia un descontento mayor que las ausencia, puesto que los beneficios de las presencias en los entornos 2.0 empiezan a conseguirse desde el momento en que se elimina la unidireccionalidad, surge el Feedback y la comunicación se vuelve bidireccional. (2011:5).

Por otra parte y como un aporte al debate sobre las ventajas que ofrece el usos de las redes sociales en las campañas electorales, D'Adamo et al., considera que se establece una especie de " "coproducción", en tanto que los votantes pueden subir fotos, compartir o aportar contenidos, "postear" o "retuitear" (...), da lugar a lo que hemos denominado "narrativas políticas transmedia" que definimos como la posibilidad de expandir el mensaje o la historia política" (2015: 109-110). Como vemos dicho proceso permite que el mensaje llegue a una mayor cantidad de usuarios. 
Antes de concluir este apartado, tenemos que reconocer que "en esta esfera virtual las redes sociales se erigen como un recurso y canal adecuado para la implementación del marketing político 2.0, creando un escenario donde es posible la interacción con los usuarios" (Túñez y Sixto, 2011: 1).

\section{Materiales y metodología}

En cuanto a la comunicación política electoral se refiere, hay que reconocer las dificultades que se presentan a la hora de realizar una campaña electoral dominicana desde el exterior, ya que el nivel de vida y las necesidades de los dominicanos residentes en el extranjero presentan ciertas diferencias en cuanto a calidad se refiere, y el espacio geográfico de las circunscripciones electorales es diverso y muy amplio porque abarca varios Estados, y las normas de cada país son diferentes en cuanto a publicidad se refiere, lo que genera limitaciones para realizar actividades proselitistas. Por lo expuesto, existen grandes diferencias en la forma de realizar una campaña electoral en el exterior, respecto a los procedimientos que se usan en República Dominicana.

Siguiendo esos aspectos, con relación a la comunicación política, la creación de las circunscripciones electorales dominicanas extraterritoriales supuso una transformación en la forma de desarrollar la campaña electoral desde el exterior, entre otras cuestiones por lo difícil de establecer contacto con los votantes desde los diversos países que las conforman.

De ahí nuestra inclinación en analizar el uso que dieron los candidatos de ultramar a las TIC, Internet y redes sociales. Ya que gracias al uso de estas herramientas, los políticos pueden lanzar sus campañas digitales y llegar a los diferentes tipos de públicos de forma eficaz, ahora bien, el resultado efectivo o no dependerá de la estrategia utilizada en cada plataforma digital.

Nuestro objetivo general estuvo delimitado en conocer el uso de las TIC, Internet y las redes sociales que realizaron los candidatos a diputados de ultramar dominicanos durante la campaña electoral. 
Uso de las TIC, Internet y redes sociales durante la campaña electoral exterior... | 11

ARTÍCULOS DE INVESTIGACIÓN

La población o universo objeto estudio corresponden al máximo Responsable de Comunicación de los 98 candidatos pertenecientes a los 14 partidos que participaron en la contienda electoral dentro de la tres circunscripción (la primera circunscripción correspondiente a Canadá y Estados Unidos, donde se debían elegir 3 diputados; la segunda comprendía parte de El Caribe, Latinoamérica y Miami, donde se elegirían 2 diputados; y por último la tercera correspondiente a Europa, donde resultarían electos 2 diputados). Se utilizó la técnica de la encuesta. Para aplicar la encuesta con las variables necesarias que daban respuesta a nuestro objetivo, se seleccionó una muestra final de 15 responsables de Comunicación.

El procedimiento de muestreo en este análisis abarca dos modalidades diferentes: primero, se seleccionaron para enviar la encuesta a los siete candidatos que resultaron electos en el proceso de las elecciones, correspondiente a las fuerzas políticas del Partido Revolucionario Dominicano (PRD) y al Partido de la Liberación Dominicana (PLD), luego a todos los candidatos que acompañaban a estos en la lista del partido, debido a que el citado conjunto presentaba más posibilidades de obtener un escaño. Finalmente también incluimos a los candidatos de partidos con menos posibilidad de ganar.

Decidimos incluir en la selección de contacto para la muestra, al menos una representación de cada partido que participó, esos candidatos se seleccionaron de acuerdo al orden de la boleta electoral, con los cuales se intentó establecer contacto.

A los candidatos de los 14 partidos que conformaron la muestra se les localizó por diferentes vías (correo electrónico, red social Facebook, teléfono, y de forma personal), con el objetivo de que nos pusieran en contacto con sus encargados de Comunicación. De forma aleatoria obtuvimos una muestra de 15 encargados de Comunicación de candidatos pertenecientes a seis partidos de las 14 fuerzas políticas que participaron en el proceso. Siete pertenecen a la totalidad de diputados que resultaron electos y ocho a los candidatos no electos que participaron en las elecciones.

Por la complejidad que presenta que un encargado de Comunicación de un candidato a diputado de ultramar ganador de la contienda electoral, acceda hablar de cuestiones estratégicas de la comunicación electoral utilizada, decidimos primero 
ponernos en contacto de manera personal con los siete diputados que resultaron electos. Por lo que tuvimos que trasladarnos desde Madrid, España a la República Dominicana, ya que es desde el Congreso dominicano desde donde desempeñan principalmente su tarea.

Durante tres meses desde República Dominicana (noviembre y diciembre del 2014; y enero del 2015), primero hicimos contactos con la secretaria de cada diputado para concertar una "entrevista-reunión" con los legisladores ultramarinos, de manera que pudiéramos contar el objetivo del estudio y así obtener su confianza para que autorizaran a sus encargados de Comunicación a rellenarnos la encuesta.

Las "entrevistas reuniones" fueron utilizadas como modo de captación de contactos y no fueron consideradas material de análisis. Una vez conseguido los contactos de los encargados de Comunicación de los diputados, conectamos con ellos vía teléfonos en algunos casos y por correos electrónicos en otros casos. Luego se les envió una carta de solicitud en la que se les explicaba que nos habíamos reunidos con los diputados de ultramar electos y que nos habían autorizado a realizar el envío de la encuesta, para que como encargado de Comunicación durante la campaña efectuaran el rellenado.

Para el caso de los candidatos que no resultaron ganadores, el proceso fue similar, pero con la diferencia de que no se realizó la "entrevista-reunión" para que facilitaran el contacto del encargado de Comunicación, sino que se les solicitó vía telefónica, por correos electrónicos o redes sociales.

La encuesta aplicada fue creada a través del programa "Formularios de Google". El cuestionario estuvo compuesto por preguntas cerradas y abiertas. Según la clasificación de preguntas que expone Vinuesa (2005), las modalidades que contiene el presente estudio se agrupan en preguntas tipo filtro, de control, de introducción o rompehielos, amortiguadoras, de opinión, tipo test basadas en la memoria.

Se utilizó la técnica de investigación de la encuesta, ya que a través de ella podemos "cuantificar variables, es decir, para obtener una información muy variada en cuanto a la clasificación del colectivo, su nivel de conocimiento sobre la 
Uso de las TIC, Internet y redes sociales durante la campaña electoral exterior... 13 ARTÍCULOS DE INVESTIGACIÓN

realidad social, sus valores, opiniones, motivaciones, expectativas, y actitudes" (Alguacil, 2011: 65).

Aunque en este artículo solo presentaremos las cuestiones relacionadas con el uso de las TIC, Internet, y redes sociales, el cuestionario quedó diseñado con un total de 36 preguntas, las cuales se agruparon en 5 categorías, veamos:

1. Indicadores relacionados con los datos generales del encargado de Comunicación y del candidato.

2. Indicadores relacionados al tipo de campaña de comunicación política electoral.

3. Indicadores relacionados con las técnicas estratégicas de comunicación política electoral vía los medios de comunicación, redes sociales, y canales alternos tradicionales (posters o vallas publicitarias, carteles, etc.).

4. Indicadores relacionados con las actividades políticas durante la campaña, el mensaje y el programa electoral.

5. Indicadores relacionados con la evaluación final sobre la cobertura general y las técnicas estratégicas de comunicación política electoral utilizada.

Posteriormente se procedió a enviar la encuesta vía correo electrónico a cada encargado de Comunicación y en algunos casos a ciertos candidatos que luego la remitirían al encargado, en otros casos ellos mismos hicieron la labor de Comunicación, al no tener una persona específica contratada o no para dicha función.

Una vez obtenida las respuestas de las 15 encuestas y su posterior codificación, pusimos en práctica lo que recoge Vinuesa, cuando se refiere que en la interpretación de resultados "las respuestas se agrupan y cuantifican para posteriormente, examinar mediante análisis estadísticos las relaciones entre ellas (Cea d' Ancona, 1998: 240-241)" (en Vinuesa, 2005: 181). 
El análisis de las preguntas se realizó vía el sistema informático que permite analizar datos (SPSS), utilizamos la estadística descriptiva con los análisis de frecuencias ejecutados. Se confeccionó un libro de códigos, para una mejor presentación de los resultados. 0 . R. Holsti describe la codificación como "el proceso por el que los datos brutos son transformados sistemáticamente y agregados en unidades que permiten una descripción precisa de las características pertinentes del contenido" (citado por Bardin, 2002: 78). Con referencia a las encuestas realizadas, se efectuó un análisis de tipo cuantitativo.

\section{Resultados}

A continuación presentamos los resultados más relevantes obtenidos de las encuestas realizadas a los encargados de Comunicación de los candidatos a diputados que participaron en las elecciones de los diputados de ultramar de la República Dominicana del 2012. Aquí destacamos el uso de las TIC, Internet y las redes sociales que realizaron durante la campaña los aspirantes.

\section{Medios publicitarios e inversión}

\begin{tabular}{|c|c|c|c|}
\hline \multirow{2}{*}{ Medios publicitarios } & \multicolumn{2}{|c|}{ Respuestas } & \multirow{2}{*}{ Porcentaje de casos } \\
\cline { 2 - 3 } & $\mathrm{N}^{\circ}$ & Porcentaje & \\
\hline Posters o vallas publicitarias & 9 & $12,9 \%$ & $60,0 \%$ \\
\hline Carteles publicitarios & 9 & $12,9 \%$ & $60,0 \%$ \\
\hline $\begin{array}{c}\text { Inserciones (anuncios) publicitarios en } \\
\text { prensa }\end{array}$ & 10 & $14,3 \%$ & $66,7 \%$ \\
\hline $\begin{array}{c}\text { Inserciones (anuncios) publicitarios en } \\
\text { Radio }\end{array}$ & 11 & $15,7 \%$ & $73,3 \%$ \\
\hline $\begin{array}{c}\text { Inserciones (anuncios) publicitarios en } \\
\text { televisión }\end{array}$ & 10 & $14,3 \%$ & $66,7 \%$ \\
\hline Octavillas & 1 & $1,4 \%$ & $6,7 \%$ \\
\hline Internet & 13 & $18,6 \%$ & $86,7 \%$ \\
\hline $\begin{array}{c}\text { Envío de cartas o correos electrónicos a } \\
\text { los electores }\end{array}$ & 4 & $5,7 \%$ & $26,7 \%$ \\
\hline
\end{tabular}


Uso de las TIC, Internet y redes sociales durante la campaña electoral exterior... | 15 ARTÍCULOS DE INVESTIGACIÓN

\begin{tabular}{|c|c|c|c|}
\hline \multirow{2}{*}{ Medios publicitarios } & \multicolumn{2}{|c|}{ Respuestas } & \multirow{2}{*}{ Porcentaje de casos } \\
\cline { 2 - 3 } & $\mathbf{N}^{\mathbf{0}}$ & Porcentaje & \\
\hline Otros & 3 & $4,3 \%$ & $20,0 \%$ \\
\hline Total & 70 & $100,0 \%$ & $466,7 \%$ \\
\hline
\end{tabular}

Tabla 2: Medios publicitarios. Fuente: elaboración propia.

En la tabla 2 aparecen los resultados de frecuencias con los que respondieron los encargados de Comunicación de los candidatos, al indicársele que seleccionaran los medios publicitarios que se utilizaron en la campaña, en esta pregunta tenían la opción de elegir todos los medios que utilizaron para insertar publicidad. Como vemos el más común utilizado por los aspirantes de ultramar, fue la publicidad por Internet, ya que de los 15 encuestados, 13 coincidieron en utilizar este formato. En segundo lugar, 11 escogieron los anuncios publicitarios en radio. En tanto que en tercer lugar, 10 también colocaron anuncios tanto en prensa como en televisión.

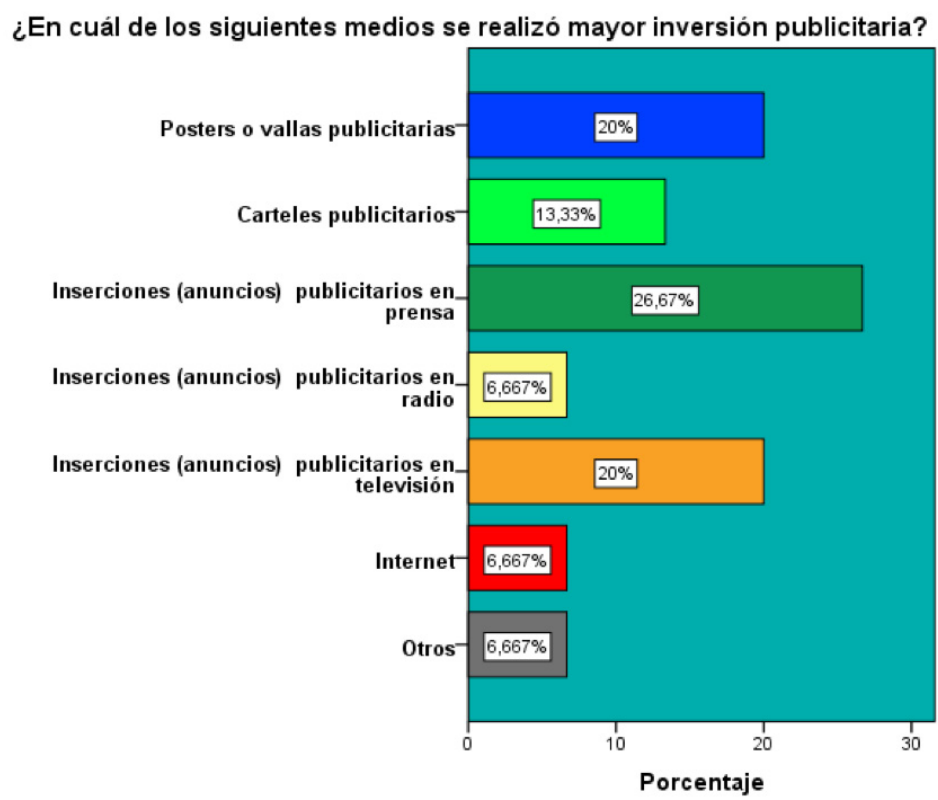

Figura 1: Fuente: elaboración propia. 
Con respecto a la inversión publicitaria durante la campaña electoral, para llevar el mensaje electoral a los electores, se les preguntó en qué medio realizaron una mayor inversión publicitaria.

En la figura 1, vemos que los resultados arrojaron que en primer lugar, un $26,7 \%$ de los candidatos dedicó una mayor inversión en los anuncios publicitarios en prensa. En segundo lugar, aparece que un $20 \%$ de los aspirantes se anunciaron mediante posters o vallas publicitarias, al igual que en la televisión, que también ocupó un $20 \%$. Un $13 \%$ invirtió más en carteles publicitarios. Por último, un $6,7 \%$ prefirió hacerlo en Internet y en radio.

La verdad es que algunos resultados nos sorprenden un poco, visto el tipo de elecciones que fueron, entendemos como lógico y normal que una mayor cantidad de la que aparece prefiriera invertir más en anunciarse vía prensa o por Internet. Pero lo que nos parece apático es que un $20 \%$ optara por la televisión, lo que nos despierta la interrogante, de que para ser unas elecciones desde el extranjero, donde los canales de televisión están regulados para pasar publicidad electoral, incluso para sus propios candidatos, entonces. ¿En qué tipo de televisión se anunciarían los candidatos de ultramar? ¿Sería que se referían a canales por Internet? ¿ 0 en su defecto ese $20 \%$ no fueron sinceros al responder? En un futuro estudio indagaremos más sobre la variable en cuestión.

\section{Envío de notas de prensa a diarios digitales}

\begin{tabular}{|c|c|c|c|}
\hline \multirow{2}{*}{$\begin{array}{c}\text { Medios digitales y notas de } \\
\text { prensa }\end{array}$} & \multicolumn{2}{|c|}{ Respuestas } & \multirow{2}{*}{ Porcentaje de casos } \\
\cline { 2 - 3 } & $\mathbf{N}^{\mathbf{0}}$ & Porcentaje & \\
\hline Listín Diario & 6 & $13,0 \%$ & $42,9 \%$ \\
\hline Diario Libre & 6 & $13,0 \%$ & $42,9 \%$ \\
\hline El Nacional & 8 & $17,4 \%$ & $57,1 \%$ \\
\hline Hoy & 6 & $13,0 \%$ & $42,9 \%$ \\
\hline El Caribe & 4 & $8,7 \%$ & $28,6 \%$ \\
\hline El Nuevo Diario & 12 & $26,1 \%$ & $85,7 \%$ \\
\hline
\end{tabular}


Uso de las TIC, Internet y redes sociales durante la campaña electoral exterior... | 17

ARTÍCULOS DE INVESTIGACIÓN

\begin{tabular}{|c|c|c|c|}
\hline \multirow{2}{*}{$\begin{array}{c}\text { Medios digitales y notas de } \\
\text { prensa }\end{array}$} & \multicolumn{2}{|c|}{ Respuestas } & \multirow{2}{*}{ Porcentaje de casos } \\
\cline { 2 - 3 } & $\mathbf{N}^{\mathbf{0}}$ & Porcentaje & \\
\hline El Día & 4 & $8,7 \%$ & $28,6 \%$ \\
\hline Total & 46 & $100,0 \%$ & $328,6 \%$ \\
\hline
\end{tabular}

Tabla 3: Medios digitales y notas de prensa. Fuente: elaboración propia.

Con relación a los medios de comunicación digitales, a los encuestados se les indicaba que seleccionaran de una lista todos los medios a los que enviaron notas de prensa, por lo que tenían la opción de seleccionar varios a la vez.

Al analizar la citada respuesta múltiple, según vemos en la tabla 3, en primer lugar, 12 candidatos coincidieron en enviar notas de prensa al periódico $E l$ nuevo Diario, nos resulta paradójico, ya que el citado medio no se encontraba entre los primeros lugares según el número de visitas durante la campaña electoral. En segundo lugar, 8 candidaturas coincidieron en enviar notas de prensa al periódico El Nacional, quien ocupaba la tercera posición en cuanto a visitas se refiere en los últimos 5 meses previos a la campaña. Tercero, 6 candidaturas enviaron notas de prensa tanto al periódico Listín Diario como al Diario Libre, quienes ocupaban la primera y segundan posición en el ranking de visitas respectivamente.

A continuación el siguiente gráfico (ver figura 2), observamos que durante la campaña electoral el $80 \%$ de las candidaturas utilizó ya sea una página web o un blog, es decir que hicieron uso de dicha herramienta para mantener a sus votantes informados. El 20\% restante afirmó no haber utilizado ni páginas webs, ni blogs, a pesar de la importancia que viene representado en los últimos años que un candidato mantenga presencia en el ecosistema digital durante la campaña electoral. Por lo que resulta de vital importancia sostener al menos unas de las dos herramientas (página web o blog). 


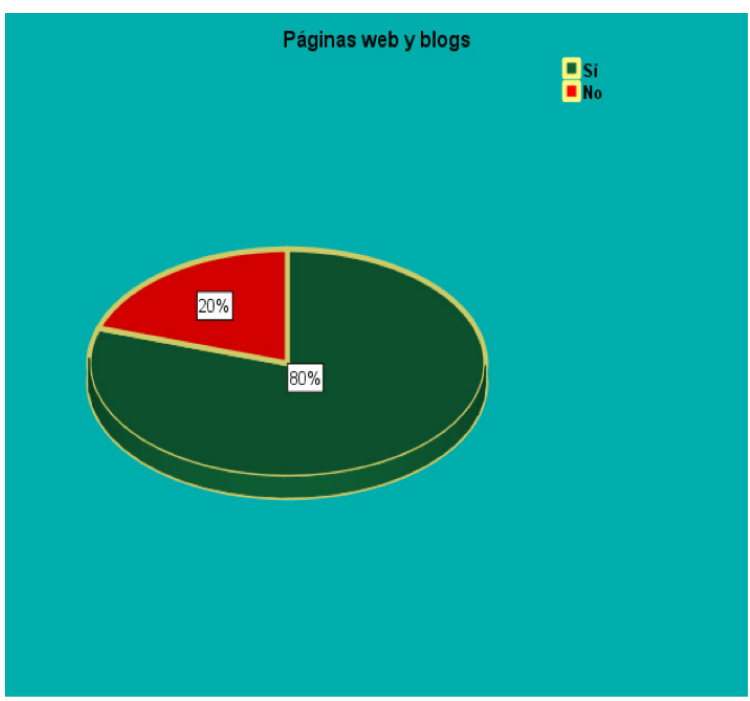

Figura 2: Fuente: elaboración propia.

\section{Redes sociales}

En la siguiente tabla número 4, analizamos el uso de las redes sociales. En la encuesta se les pedía a los encargados de Comunicación que seleccionaran de una lista de redes sociales, todas en las que el candidato tenía cuenta, por lo que tenían la opción de seleccionar varias a la vez, y por último tenían habilitada una casilla para indicar que no, esto en caso de que no tuvieran ninguna cuenta.

Los resultados arrojaron que de los 15 candidatos, 14 tenían cuentas en algunas de las redes sociales, es decir, el 93,3\% de los encuestados, excepto un candidato que resultó electo, y afirmó que no tenía encargado de Comunicación.

\begin{tabular}{|c|c|c|c|}
\hline \multirow{2}{*}{ Redes Sociales } & \multicolumn{2}{|c|}{ Respuestas } & \multirow{2}{*}{ Porcentaje de casos } \\
\cline { 2 - 4 } & No & Porcentaje & \\
\hline Facebook & 14 & $40,0 \%$ & $93,3 \%$ \\
\hline Twitter & 6 & $17,1 \%$ & $40,0 \%$ \\
\hline YouTube & 7 & $20,0 \%$ & $46,7 \%$ \\
\hline Google + & 5 & $14,3 \%$ & $33,3 \%$ \\
\hline
\end{tabular}


Uso de las TIC, Internet y redes sociales durante la campaña electoral exterior... | 19

ARTÍCULOS DE INVESTIGACIÓN

\begin{tabular}{|c|c|c|c|}
\hline \multirow{2}{*}{ Redes Sociales } & \multicolumn{2}{|c|}{ Respuestas } & \multirow{2}{*}{ Porcentaje de casos } \\
\cline { 2 - 3 } & $\mathbf{N}^{\mathbf{0}}$ & Porcentaje & \\
\hline Instagram & 2 & $5,7 \%$ & $13,3 \%$ \\
\hline No tenía & 1 & $2,9 \%$ & $6,7 \%$ \\
\hline Total & 35 & $100,0 \%$ & $233,3 \%$ \\
\hline
\end{tabular}

Tabla 4: Redes sociales. Fuente: elaboración propia.

Normalmente las dos redes sociales más utilizadas en las campañas electorales suelen ser Facebook y Twitter. En una entrevista para EFE, José Luis Orihuela, profesor de Comunicación de la Universidad de Navarra, declaró que, "los blogs, Facebook y Twitter se han convertido en «el trípode» sobre el que se asienta cualquier estrategia de comunicación en Internet (...) permiten un mayor grado de acercamiento entre los candidatos y los ciudadanos" (Viar, 2011). Pero para las elecciones de los diputados de ultramar, vemos que fue todo lo contrario. Los candidatos prefirieron usar en primer y segundo lugar Facebook y YouTube, y en tercer lugar Twitter.

Con respecto a la valoración de las redes sociales, (ver figura 3 y 4) la mejor valorada por las candidaturas fue Facebook, de las 15, 10 coincidieron en reconocer que es muy importante. En cambio la peor valorada fue LinkedIn, ya que de las ocho que la valoraron, todas coincidieron en indicar que fue "nada importante" en la estrategia de su comunicación electoral vía las redes sociales.

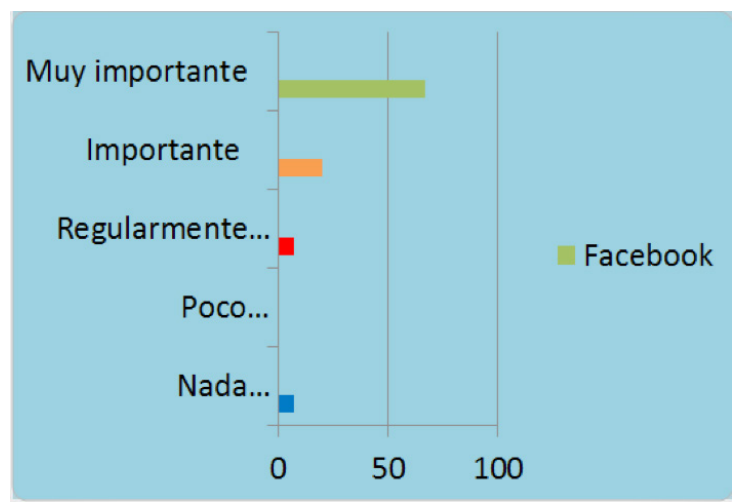

Figura 3: Fuente: elaboración propia. 


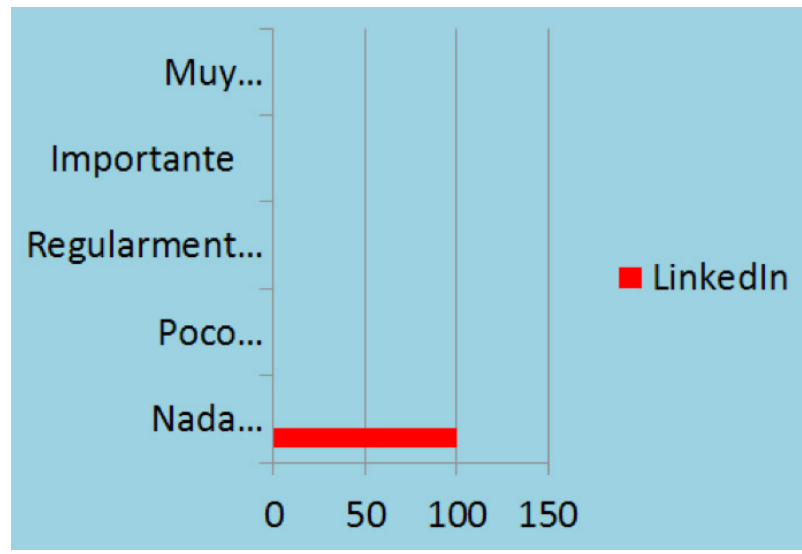

Figura 4: Fuente: elaboración propia.

\section{Conclusiones y discusión}

Después de analizar los resultados obtenidos en nuestro estudio de investigación de las encuestas que aplicamos a 15 encargados de Comunicación de candidatos que participaron en las elecciones de los diputados de ultramar de la República Dominicana del año 2012, llegamos a las siguientes conclusiones y discusión.

Con relación a los medios publicitarios que se utilizaron en la campaña, a nivel de frecuencias quedó demostrado que el más común utilizado por los aspirantes de ultramar, fue la publicidad por internet, ya que de los 15 encuestados, 13 coincidieron en utilizar este formato. Aunque la mayoría de los candidatos $(26,7 \%)$ destinaron una mayor inversión publicitaria a los anuncios en prensa como tradicionalmente se ha hecho.

De los siete candidatos que resultaron electos como diputados de ultramar, únicamente uno de ellos afirmó no haber tenido encargado de Comunicación y no haber realizado labores de comunicación con los medios digitales, además indicó no tener cuentas en las redes sociales.

De los 15 candidatos que participaron en el estudio, prácticamente todos enviaron notas de prensa a los medios digitales, excepto el que no realizó labores de comunicación y que resaltamos anteriormente. 
A las TIC aplicadas a la comunicación política se les puede sacar mucho provecho. Por vía del correo electrónico y los blogs se puede obtener una descripción de las demandas ciudadanas $\mathrm{y}$, a partir de ahí se articula mejor el discurso de forma segmentada para cada necesidad según el público, que fue lo que hizo Barack Obama (Castro, 2012). Lo mismo podemos hacer con el uso estratégico de las redes sociales, donde los candidatos pueden interactuar con el electorado en tiempo real. Existen otras herramientas y foros a través de la red donde los electores pueden comunicarse con los candidatos e incluso dejar sus propuestas.

A pesar de la importancia que cada vez adquieren las páginas webs y los blog para los candidatos durante las campañas electorales, todavía un $20 \%$ de las candidaturas de ultramar encuestadas afirmó no haber utilizado al menos una de las dos herramientas citadas.

El 93,3\% de las candidaturas encuestadas mantenían cuentas en alguna red social (Facebook, Twitter, YouTube, Google +, o Instagram). Para las elecciones de los diputados de ultramar los candidatos prefirieron usar más Facebook y YouTube, dicho resultado nos resulta atípico, ya que durante la campaña electoral exceptuando los blogs, las estrategias de comunicación digital de los candidatos se acentúan más en Facebook y Twitter (Viar, 2011). Por otra parte descubrimos que la red social mejor valorada por las candidaturas fue Facebook y la peor valoradora LinkedIn.

Está claro que en la actualidad cada vez más los candidatos realizan un uso inteligente de Internet, las TIC y las redes sociales que están al servicio de la comunicación política. Para el caso de las campañas electorales, podríamos citar entre las más utilizadas: Facebook, Twitter, YouTube, Google +, LinkedIn, Instagram, correo electrónico, teléfono, celular, páginas webs, blog, etc., dichas herramientas son utilizadas para promover los candidatos a través del envío de mensajes y publicidad estratégica. La gran ventaja que ofrecen es que se puede segmentar mejor a los votantes, de manera que el mensaje estratégico pueda llegar a grupos de públicos específicos. Resultan gratuitas, y se da un mayor nivel de interacción con nuestros internautas. 
Por otra parte, tenemos que resaltar que ha aumentado la inversión en publicidad digital durante las campañas electorales, incluso las suelen subdividir y se les llama campañas digitales o campañas online.

La clase política ha descubierto en el fenómeno digital una vía atractiva y seductora para difundir sus mensajes, especialmente "en los periodos electorales, este novedoso escenario de información, comunicación e interacción sociopolítica se ve literalmente inundado con mensajes, fotografías, presentaciones electrónicas y videos creados y difundidos no solo por los equipos de campaña, sino por los consumidores" (Galeas de la Vega, 2012: 202).

A pesar de que no se le dio un uso estratégico, las TIC, Internet y las redes sociales sí fueron utilizadas durante la campaña electoral de las elecciones de los diputados de ultramar.

No obstante, pese a la distancia entre los países que conforman las circunscripciones extraterritoriales, lo cual añade la dificultad para mantener una relación directa con los votantes, muchos candidatos prefirieron continuar con la comunicación tradicional.

Galeas de la Vega (2012) entiende que la comunicación digital ha pasado a ser una receta ideal, segura e inmediata para que los candidatos puedan obtener una victoria electoral. Observamos que todo candidato necesita incluir en su plan estratégico la comunicación digital, pero no que su éxito electoral dependa única y exclusivamente de esta, sino, más bien agregamos que la comunicación tradicional sigue siendo aún importante y necesariamente se debe completar el plan estratégico con la comunicación digital.

Coincidimos con Dávalos (2011) en que las campañas políticas tienen que reconocer que la comunicación actual no va en una sola dirección y que de manera significativa los avances tecnológicos han marcado notablemente tanto a los medios de comunicación como a las audiencias. Por lo que se hace necesaria la adaptación de la comunicación política también al uso de los medios alternativos y tecnológicos. 
Uso de las TIC, Internet y redes sociales durante la campaña electoral exterior... | 23

ARTÍCULOS DE INVESTIGACIÓN

No queremos concluir sin antes hacer mención de las famosas vallas publicitarias o los carteles publicitarios con la imagen del candidato y los eslóganes que tanto vemos en las calles durante una campaña electoral. Pues estos también funcionan como un medio o canal alternativo, y más ahora que se adaptan a los formatos digitales. "La publicidad traslada a un formato audiovisual el discurso político, sintetizándolo en unas pocas frases que apelan a la emoción y buscan convencer al elector por un determinado candidato" (IDEA, 2006: 30).

Para concluir, reconocemos que a pesar de las ventajas que ofrecen las TIC, Internet y las redes sociales a la comunicación política, es cierto que existe una desventaja denominada brecha digital, donde existe una gran cantidad de personas en varios países del mundo que no tiene acceso a Internet y mucho menos a las nuevas tecnologías, aspecto que estudiaremos en una futura investigación.

\section{Referencias}

Alguacil Gómez, J. (2011). Cómo se hace un trabajo de investigación en Sociología. Madrid: Catarata.

Asamblea Nacional del Ecuador. (2017). Obtenido de https://www.asambleanacional. gob.ec/es

Bardin, L. (2002). Análisis de contenido (3 ed.). Madrid: Akal.

Castells, M. (2003). Internet, libertad y sociedad: una perspectiva analítica. Polis, (4), 1-20. Recuperado de https://bit.ly/2EtAUqu

Castro Martínez, L. (2012). El marketing político en Estados Unidos: el caso Obama. Norteamérica, 7(1), 209 -222. Recuperado de https://goo.gl/2jHb97

Collyer, M., \& Vathi, Z. (2007). Patterns of Extra-territorial Voting. Sussex Centre for Migration Research, Working Paper T22, 0ctober. Recuperado de https:// goo.gl/zYywxJ

D’Adamo, 0., García Beaudoux, V., Kievsky, T. (2015). Comunicación política y redes sociales: análisis de las campañas para las elecciones legislativas de 2013 en la ciudad de Buenos Aires. Revista Mexicana de Opinión Pública, 19 (juliodiciembre), 107-125. Recuperado de https://bit.ly/2LJbB3I

Dávalos López, A. (Abril de 2011). Cómo diseñar campañas políticas exitosas. En Barrera A., Alcaldía de Quito, Ivoskus D. (Organizadores), La comunicación 
política. Conferencia llevada a cabo en II Cumbre Mundial de Comunicación Política, Quito, Ecuador.

De León, V. (23 de abril de 2012). Canadá no aceptó elecciones de ultramar RD. Listín Diario. Recuperado de https://goo.gl/fdsQVH

Del Orbe Ayala, K.R. (2017). Voto exterior, una mirada desde la opinión pública: Análisis de la transformación en la representación política parlamentaria de la República Dominicana en 2012. Veredas, (35), 73-86. Recuperado de https:// bit.ly/2JhC7To

Elgarresta, M. J. (2003). Conocimientos prácticos para ganar elecciones. Recuperado de https://goo.gl/j4k5GJ

Galeas de la Vega, C. (2012). Los nuevos evangelizadores. La campaña de Obama '08 en un planeta interactivo. En Dávalos López, A., Polanco Frías, V., Galeas de la Vega, C., y Pérez, A. (Ed.), ¿Cómo diseñar campañas políticas exitosas? (pp. 201-243). Quito: CIESPAL.

García Galera, M.C., y Fernández Muñoz, C. (2016). Si lo vives, lo compartes. Cómo se comunican los jóvenes en un mundo digital. Barcelona: Ariel.

Gautreau, J. M. (Setiembre 2012). El voto en el exterior, la experiencia dominicana. En Tribunal Supremo Electoral de El Salvador, IDEA International, y el Instituto de Investigaciones Jurídicas de la UNAM (organizadores). Conferencia llevada a cabo en la IV Conferencia Iberoamericana sobre Justicia Electoral, San Salvador, El Salvador.

Gutiérrez-Rubí, A. (18 de enero de 2016). Crowdfunding y campañas. Antoni Gutiérrez-Rubi. Recuperado de https://bit.ly/2kBPFLs

IDEA. (2006). Comunicación política en campañas electorales. Módulo avanzado de formación política. Stockholm: IDEA.

Junta Central Electoral, JCE. (2012). Resultados por colegios 2012 Dip. Exterior. Santo Domingo. R.D. Recuperado de https://goo.gl/mWgsv7

Junta Central Electoral. (2013). Resultados Electorales 2012. Elecciones Ordinarias Generales Presidenciales y de Diputados y Diputadas en el Exterior del 20 de Mayo del 2012. Santo Domingo. R.D. Recuperado de https://goo.gl/FCtHxf Ley No. 136, que regula el voto de los dominicanos en el exterior, para la elección de los diputados y diputadas de ultramar, del 7 de junio de 2011. Santo Domingo. Mazzoleni, G. (2004). La comunicazione politica. Bologna: ilMulino. Trad. Linares, P. (2010). Madrid: Alianza. 
Uso de las TIC, Internet y redes sociales durante la campaña electoral exterior... | 25

ARTÍCULOS DE INVESTIGACIÓN

Mieres, P. (2 de marzo de 2015). Tendencia de la comunicación política y las campañas electorales en América Latina. ASESMAP. Recuperado de https:// goo.gl/HiiiP5

Navarro, C. (Noviembre de 2012). Panorama comparado de los retos y alternativas para la regulación del voto en el extranjero. En Organización de los Estados Americanos (OEA), Instituto Federal Electoral (IFE) e IDEA (organizadores), Voto desde el extranjero: retos y realidades y la participación política de las mujeres. Conferencia llevada a cabo en la V Jornada Interamericana Electoral, D.F., México. Navarro, C., Morales, I., \& Gratschew, M. (2007). Comparative overview of voting abroad. En Ellis, A., Navarro, C., Morales, l., Gratschew, M., \& Braun, N. (Ed), Voting from abroad: An International IDEA Handbook (pp. 11-49). Stockholm: Internacional IDEA. Trad. cast.: Instituto Federal Electoral de México. (2008). Voto en el extranjero. El manual de IDEA Internacional. México: Instituto Federal Electoral de México.

Organización de los Estados Americanos, OEA. (2013). Informe de la Misión de Observación Electoral de la OEA. Elecciones Ordinarias Generales Presidenciales del 20 de Mayo de 2012 en la República Dominicana. Washington D.C., Estados Unidos. Recuperado de https://goo.gl/UWf3Sk

Sepúlveda, W. (noviembre de 2012). El reto de convertir votos desde el extranjero en escaños. En Organización de los Estados Americanos (OEA), Instituto Federal Electoral (IFE) e IDEA (organizadores), Voto desde el extranjero: retos y realidades y la participación política de las mujeres. Conferencia llevada a cabo en la V Jornada Interamericana Electoral, D.F., México.

Túñez, M. y Sixto J. (2011). Redes sociales, política y compromiso 2.0: La comunicación de los diputados españoles en Facebook. Revista Latina de Comunicación Social, (66) 1-25. doi: 10.4185/RLCS-66-2011-930-210-246

Ureña, D. (2011). Decálogo para un candidato 2.0. Cuadernos de comunicación evoca. 4, 29-33. Recuperado de https://goo.gl/jG7aF

Vargas, Y. (19 de julio de 2011a). Dominicanos en el exterior: de la participación a la representatividad. Observatorio Político Dominicano. Recuperado de https:// goo.gl/LP25y7

Vargas, Y. (10 de noviembre de 2011b). Rumbo a las elecciones del 2012. El padrón electoral al 17 de octubre de 2011. Observatorio Político Dominicano. Recuperado de https://goo.gl/Rj4fJX 


\section{ARTÍCULOS DE INVESTIGACIÓN}

Vargas, Y. (16 de febrero de 2012). Padrón electoral 2012. En Estados Unidos hay más votantes que en 27 de las provincias del país. Observatorio Político Dominicano. Recuperado de https://goo.gl/xUEEFm

Viar, A. (16 de abril de 2011). Facebook y Twitter, herramientas no aptas para políticos oportunistas. Expasión.com. Recuperado de https://goo.gl/MyQFc

Vinuesa, M. L. (2005). La encuesta. Observación extensiva de la realidad social. En Berganza Conde, M. R., y Ruiz San Román, J.A. (Ed.), Investigar en Comunicación. Guía práctica de métodos y técnicas de investigación social en Comunicación (pp.177-205). Madrid: McGraw Hill.

\section{Notas}

[1] El presente trabajo es un fragmento de mi tesis doctoral, "Elecciones de diputados de ultramar en la República Dominicana 2012. Un análisis del tratamiento informativo que ofreció la prensa digital, las prácticas de comunicación política electoral de los candidatos y la opinión pública Especializada", correspondiente al programa "Doctorado en Política, Comunicación y Cultura de la Universidad Complutense de Madrid. Aquí presentamos solo los contenidos referentes al entorno digital, obtenido del estudio 2 sobre las técnicas de comunicación política de los candidatos a diputados de ultramar de las elecciones del 2012. 